\title{
Prevalência e fatores associados a dor dentária - estudo de base populacional com adultos e idosos do sul do Brasil
}

\author{
Prevalence and factors associated with tooth pain - a population-based study \\ with adults and elderly from southern Brazil
}

\author{
Mariana Silveira ECHEVERRIA ${ }^{a *}$ (D), Samuel Carvalho DUMITH ${ }^{\mathrm{b}}$ (i), Alexandre Emidio Ribeiro SILVA ${ }^{\mathrm{C}}$ (1) \\ aUFPel - Universidade Federal de Pelotas, Faculdade de Medicina, Programa de Pós-graduação em Epidemiologia, \\ Pelotas, RS, Brasil \\ bFURG - Universidade Federal do Rio Grande, Faculdade de Medicina, Programa de Pós-graduação em Saúde Pública, \\ Rio Grande, RS, Brasil \\ 'UFPel - Universidade Federal de Pelotas, Faculdade de Odontologia, Programa de Pós-graduação em Odontologia, \\ Pelotas, RS, Brasil
}

\begin{abstract}
Como citar: Echeverria MS, Dumith SC, Silva AER. Prevalência e fatores associados a dor dentária - estudo de base populacional com adultos e idosos do sul do Brasil. Rev Odontol UNESP. 2020;49:e20200039. https://doi.org/10.1590/18072577.03920
\end{abstract}

\begin{abstract}
Resumo
Introdução: A dor dentária, ou odontalgia, é definida como uma dor de origem orofacial, que ocorre como consequência dos problemas que afetam os dentes e as estruturas de suporte. Objetivo: 0 objetivo do presente estudo foi analisar os fatores associados à dor dentária em indivíduos com 18 anos ou mais de idade, no município de Rio Grande-RS, ao sul do Brasil. Material e método: Estudo transversal de base populacional. Foram entrevistados, no domicílio, 1.099 indivíduos, por meio de um questionário com questões fechadas. Foram obtidas informações demográficas, socioeconômicas e relativas a hábitos e comportamentos de saúde geral e bucal. 0 relato de dor dentária nos seis meses anteriores ao inquérito foi o desfecho do estudo. As análises foram baseadas em regressão de Poisson. Resultado: A prevalência de dor dentária foi de 18,0\% (IC95\% 15,6; 20,4). Ao se analisar por faixa etária, a prevalência de dor dentária foi de 23,9\% (IC95\% 20,3; 27,4), 14,0\% (IC95\% 10,6; 17,5) e 10,4\% (IC95\% 5,8; 15,0), considerando a população adulta de 20 a 39 anos e de 40 a 59 anos, e a população idosa, acima de 60 anos, respectivamente. Maiores prevalências de dor origem dentária foram em indivíduos solteiros (RP=1,50; IC95\% 1,11; 2,03), com menor escolaridade (menos de oito anos de estudo) (RP=1,62; IC95\% 1,16; 2,27) e com o hábito de ranger os dentes $(\mathrm{RP}=1,67$; IC95\% 1,18; 2,38). Já os idosos apresentaram menores prevalências de dor origem dentária, quando comparados aos indivíduos adultos ( $\mathrm{RP}=0,50$; IC95\% 0,32; 0,80). Conclusão: Maior atenção deve ser dada aos solteiros, menos escolarizados, que rangem os dentes, e aos adultos jovens.

Descritores: Epidemiologia; saúde bucal; dor; adulto; idosos.
\end{abstract}

\begin{abstract}
Introduction: Dental pain, or toothache, is defined as pain of orofacial origin, which occurs as a consequence of problems that affect teeth and support structures. Objective: The objective of the present study was to analyze the factors associated with dental pain in individuals aged 18 years or older in the city of Rio Grande, RS, in the south of Brazil. Material and method: Cross-sectional population-based study. 1,099 individuals were interviewed at home using a questionnaire with closed questions. Demographic, socioeconomic, general and oral health habits and behaviors were obtained. The report of dental pain in the 6 months prior to the survey was the study's outcome. The analyzes were based on Poisson regression. Result: The prevalence of dental pain was $18.0 \%$ (95\% CI 15.6; 20.4). When analyzing by age group, the prevalence of dental pain was $23.9 \%$ (95\% CI 20.3; 27.4), $14.0 \%$ (95\% CI 10.6; 17.5) and $10.4 \%$ (95\% CI 5.8; 15.0), considering the adult population aged 20 to 39 years old, 40 to 59 years old and elderly, respectively. The highest prevalence of dental pain was for single individuals ( $P R=1.50 ; 95 \%$ CI $1.11 ; 2.03$ ), with less education (less than 8 years of study) (PR $=1.62 ; 95 \%$ CI 1.16; 2.27) and with the habit of grinding
\end{abstract}


the teeth (PR $=1.67 ; 95 \%$ CI 1.18; 2.38). The elderly, on the other hand, had lower prevalence of dental pain when compared to adult individuals (PR $=0.50 ; 95 \%$ CI $0.32 ; 0.80)$. Conclusion: Greater attention should be given to single, less educated, who grind their teeth and young adults.

Descriptors: Epidemiology; oral health; pain; adult; elderly.

\section{INTRODUÇÃO}

A dor dentária, ou odontalgia, é definida como uma dor de origem orofacial, que ocorre como consequência dos problemas que afetam os dentes e as estruturas de suporte. Os principais agravos de saúde bucal que levam a episódios de dor de dente são a cárie, as doenças periodontais e o traumatismo dentário ${ }^{1}$.

No Brasil, a prevalência de dor de dente medida pela Pesquisa Nacional de Saúde Bucal - SB Brasil 2010 foi de 27,5\% e 10,8\%, para os grupos etários de 35 a 44 e 65 a 74 anos, respectivamente, e não se demonstraram diferenças significativas entre as regiões do país ${ }^{2}$.

A presença de dor dentária pode ter implicações, como diminuição das horas de sono, prejuízo de atividades de lazer, restrições alimentares e desordens psicológicas relacionadas com a diminuição da autoestima e o absenteísmo escolar e/ou no trabalho ${ }^{3,4}$. Além disso, a dor dentária, por ser a repercussão mais impactante das doenças bucais ${ }^{5}$ e devido à sua alta prevalência ${ }^{2}$, pode ser considerada um problema de saúde pública.

Os estudos que avaliaram dor dentária entre adultos e idosos têm apontado que as maiores prevalências estão entre os indivíduos de cor preta e parda, com baixa escolaridade, que não possuem plano de saúde, que são tabagistas, consumidores de refrigerantes, que visitaram ocasionalmente um dentista e cuja última consulta odontológica foi motivada por problemas ${ }^{6,7}$. Por outro lado, idosos, aqueles com status econômico elevado, comportamento bom ou moderado de saúde bucal, e boa autopercepção de saúde bucal foram menos propensos a relatar dor dentária8.

Diante do exposto, realizar intervenções adequadas para diminuir o impacto desse problema de saúde pública na rotina das pessoas é necessário. Para tanto, é preciso conhecer a extensão do problema, ou seja, a prevalência da dor dentária e em quais grupos ela ocorre mais comumente. Portanto, o presente estudo de base populacional tem como objetivo analisar os fatores associados à dor dentária entre indivíduos maiores de idade residentes no município do Rio Grande-RS, no sul do Brasil.

\section{MATERIAL E MÉTODO}

Rio Grande é um município situado no extremo sul do estado do Rio Grande do Sul, Brasil com aproximadamente 200 mil habitantes, sendo que a maioria, cerca de $95 \%$, reside na zona urbana 9 . Neste contexto, foi realizado um consórcio de pesquisa, denominado "Saúde da População Riograndina”, entre os Programas de Pós-graduação Ciências da Saúde (PPGCS) e Saúde Pública (PPGSP) da Universidade Federal do Rio Grande (FURG), com o intuito de unir esforços para obter informações sobre o processo saúde-doença na população estudada.

Para o presente estudo, foram utilizados os dados coletados, através desse consórcio de pesquisa, de 1.099 indivíduos maiores de idade residentes na área urbana de Rio Grande-RS, em 2016, dentre os 1.300 adultos que participaram da pesquisa. Para analisar o desfecho do presente estudo, foram excluídos da amostra usuários de prótese total $(n=194)$ e sem informação $(n=7)$ para dor de dente. Dessa forma, a amostra analisada para este estudo foi constituída por 1.099 indivíduos. Este estudo apresenta delineamento transversal de base populacional. As informações sobre os participantes foram obtidas pela aplicação, por entrevistadores treinados, de um questionário estruturado com variáveis demográficas, socioeconômicas, de saúde geral e saúde bucal, no próprio domicílio do entrevistado. Informações metodológicas relacionadas ao 
cálculo amostral e a amostragem realizada para seleção dos participantes da pesquisa, entre outros aspectos, podem ser avaliadas em estudo prévio ${ }^{10}$.

A dor de dente foi a variável dependente considerada neste estudo, medida através da seguinte pergunta: "Nos últimos seis meses, isto é, desde <MÊS> até agora, o(a) Sr.(a) teve dor de dente?", com as opções de resposta: não, sim e não sabe.

As variáveis independentes avaliadas foram as características demográficas: sexo (masculino e feminino), faixa etária (20 a 39; 40 a 59; 60 anos ou mais), cor (branca e outras) e estado civil (solteiro; casado, separado ou viúvo). Para medir o nível socioeconômico, foram avaliadas a escolaridade em anos de estudo ( 0 a 8; 9 a 11; 12 anos ou mais) e o índice de bens em tercis (1, 2 e 3). Em relação aos comportamentos de saúde, foram perguntadas questões referentes ao tabagismo (não fumante; exfumante; fumante); consumo excessivo de álcool (não e sim); consumo de doces em cinco ou mais dias da semana (não e sim), e consumo regular de refrigerantes em cinco ou mais dias da semana (não e sim). Por fim, as variáveis sobre a saúde bucal obtidas dos participantes foram: frequência de escovação diária (até duas e três ou mais vezes), tipo de serviço usado na última consulta (público; particular; convênio), ranger os dentes (não e sim) e uso de prótese parcial (não e sim). Foram excluídos do estudo indivíduos usuários de prótese total.

As análises estatísticas foram feitas no programa estatístico Stata, versão 15.0. Foram realizadas inicialmente análises descritivas. Posteriormente, foi usada a regressão de Poisson, com ajuste robusto de variância, para a análise bruta e ajustada. As medidas foram expressas por meio de razão de prevalência (RP) e intervalo de confiança de 95\% (IC95\%). Para a análise ajustada, construiu-se um modelo hierárquico em três níveis, considerando, no primeiro nível, as variáveis demográficas e socioeconômicas; no segundo nível, as variáveis comportamentais, e no terceiro nível, as variáveis relacionadas à saúde bucal. Foi usado ajuste com regressão para trás, em que as variáveis foram controladas para aquelas do mesmo nível ou de níveis acima. Todas as variáveis foram levadas para o modelo ajustado e o nível de significância para manutenção das variáveis foi $p<0,20$. Para a análise dos possíveis fatores associados com dor de dente, a análise foi ajustada para todas as variáveis que apresentaram $\mathrm{p}<0,20$. 0 nível de significância estatística adotado foi de $5 \%$.

O projeto de pesquisa foi aprovado pelo Comitê de Ética em Pesquisa na Área da Saúde da FURG em março de 2016 (parecer 20/2016). Todos os participantes do estudo assinaram um Termo de Consentimento Livre e Esclarecido (TCLE) para participar da pesquisa. Os indivíduos que não sabiam ler e/ou escrever consentiram em participar colocando sua impressão digital no termo.

\section{RESULTADO}

A prevalência de dor dentária foi de 23,9\% (IC95\% 20,3; 27,4), 14,0\% (IC95\% 10,6; 17,5) e $10,4 \%$ (IC95\% 5,8; 15,0), considerando a população adulta de 20 a 39 anos e de 40 a 59 anos, e a população idosa, acima de 60 anos, respectivamente. Na amostra como um todo, a prevalência de dor dentária foi de $18 \% \operatorname{IC} 95 \%(15,6 ; 20,4)$. A maior parte da amostra era constituída por mulheres (55,3\%), com idade entre 20 e 39 anos $(46,2 \%)$, cor branca $(82,8 \%)$, estado civil solteiro $(51,3 \%)$ e com até oito anos de estudo $(36,4 \%)$. Quanto aos comportamentos de saúde, a maioria relatou não utilizar tabaco $(58,8 \%)$ e nem consumir álcool em excesso $(87,1 \%)$. Para o consumo de doces, 69,4\% da amostra não ingeriu em mais de cinco dias por semana, já para o refrigerante, o percentual de não consumo foi de $54,2 \%$. Em relação à rotina diária de hábitos de higiene bucal, a maior parte dos entrevistados escovava mais de três vezes por dia $(65,1 \%)$ e não usava o fio dental $(70,6 \%)$. Além disso, $72,1 \%$ da amostra consultou-se com um dentista no último ano e o tipo de serviço odontológico mais usado na última consulta foi o particular (52,4\%). Por fim, 10,7\% relataram ranger os dentes e 18,5\% utilizavam alguma prótese parcial. (Tabela 1). 
Tabela 1. Descrição da população adulta e idosa do município de Rio Grande-RS, 2016 ( $N=1.099)$

\begin{tabular}{|c|c|c|}
\hline Variável & $\mathbf{N}$ & $\%$ \\
\hline \multicolumn{3}{|l|}{ Sexo } \\
\hline Masculino & 491 & 44,7 \\
\hline Feminino & 608 & 55,3 \\
\hline \multicolumn{3}{|l|}{ Faixa etária } \\
\hline $20-39$ & 507 & 46,2 \\
\hline $40-59$ & 428 & 38,9 \\
\hline 60 ou + & 164 & 14,9 \\
\hline \multicolumn{3}{|l|}{ Cor } \\
\hline Branca & 908 & 82,8 \\
\hline Outras & 189 & 17,2 \\
\hline \multicolumn{3}{|l|}{ Estado civil } \\
\hline Solteiro & 564 & 51,3 \\
\hline Casado, separado, viúvo & 535 & 48,7 \\
\hline \multicolumn{3}{|l|}{ Escolaridade (anos) } \\
\hline 0 a 8 & 400 & 36,4 \\
\hline 9 a 11 & 352 & 32,1 \\
\hline$\geq 12$ & 345 & 31,5 \\
\hline \multicolumn{3}{|l|}{ Índice de bens (tercis) } \\
\hline 1 (menor) & 367 & 33,4 \\
\hline 2 & 357 & 32,5 \\
\hline 3 (maior) & 375 & 34,1 \\
\hline \multicolumn{3}{|l|}{ Tabagismo } \\
\hline Não fumante & 646 & 58,8 \\
\hline Ex-fumante & 252 & 22,9 \\
\hline Fumante & 201 & 18,3 \\
\hline \multicolumn{3}{|l|}{ Consumo excessivo de álcool } \\
\hline Não & 955 & 87,1 \\
\hline Sim & 142 & 12,9 \\
\hline \multicolumn{3}{|c|}{ Consumo de doces ( $\geq 5$ dias por semana) } \\
\hline Não & 762 & 69,4 \\
\hline Sim & 336 & 30,6 \\
\hline \multicolumn{3}{|c|}{ Consumo de refrigerantes ( $\geq 5$ dias por semana) } \\
\hline Não & 596 & 54,2 \\
\hline Sim & 503 & 45,8 \\
\hline \multicolumn{3}{|c|}{ Número de vezes que escova os dentes por dia } \\
\hline Até 3 & 801 & 72,9 \\
\hline$\geq 4$ & 298 & 27,1 \\
\hline \multicolumn{3}{|c|}{ Consulta ao dentista no último ano } \\
\hline Não & 302 & 27,9 \\
\hline Sim & 782 & 72,1 \\
\hline \multicolumn{3}{|c|}{ Tipo de serviço usado na última consulta } \\
\hline Público & 203 & 19,4 \\
\hline Particular & 550 & 52,4 \\
\hline Convênio & 296 & 28,2 \\
\hline \multicolumn{3}{|l|}{ Range os dentes } \\
\hline Não & 970 & 89,3 \\
\hline Sim & 116 & 10,7 \\
\hline \multicolumn{3}{|l|}{ Uso de prótese } \\
\hline Não & 896 & 81,5 \\
\hline Sim (parcial) & 203 & 18,5 \\
\hline \multicolumn{3}{|c|}{ N: Número de participantes. } \\
\hline
\end{tabular}


As prevalências de dor dentária de acordo com as variáveis investigadas e os resultados da análise por regressão de Poisson bruta e ajustada podem ser observadas na Tabela 2. Os fatores associados na análise ajustada foram a faixa etária, o estado civil, a escolaridade e o hábito de ranger os dentes. A faixa etária foi associada ao menor relato de dor dentária nos últimos seis meses, em que ter entre 40 e 59 anos de idade fornece uma proteção para dor de dente $(\mathrm{RP}=0,65$; IC95\% 0,47-0,89), quando comparado aos indivíduos entre 20 e 39 anos. Além disso, idosos apresentam uma proteção ainda maior, correspondente a uma prevalência $50 \%$ menor se comparados aos adultos de 20 a 39 anos. A prevalência de dor dentária foi $50 \%$ maior entre os solteiros, comparados aos participantes casados, separados ou viúvos. Em relação à escolaridade, aqueles com até oito anos de estudo tiveram uma prevalência $62 \%$ maior de dor dentária quando comparados aos que estudaram 12 anos ou mais. Indivíduos que têm o hábito de ranger os dentes apresentaram uma prevalência $67 \%$ maior de dor dentária em relação aos que não tinham esse hábito. Ainda, vale destacar que houve uma associação limítrofe da dor de dente com o consumo de refrigerantes mesmo após o ajuste, em que a prevalência de dor dentária foi 30\% maior $(\mathrm{RP}=1,30$; IC95\% 0,$99 ; 1,71)$ entre os consumidores regulares de refrigerantes, comparados àqueles que não consomem regularmente.

Tabela 2. Análise bruta e ajustada da prevalência de dor de dente nos últimos seis meses de acordo com as características investigadas. Rio Grande-RS, $2016(\mathrm{~N}=1.099)$

\begin{tabular}{|c|c|c|c|c|}
\hline \multirow{2}{*}{ Nível } & \multirow{2}{*}{ Variável } & \multirow{2}{*}{$\begin{array}{c}\text { Dor de dente } \\
(\%)\end{array}$} & \multirow{2}{*}{$\begin{array}{c}\text { Análise bruta } \\
\text { RP (IC95\%) } \\
\end{array}$} & \multirow{2}{*}{$\begin{array}{c}\text { Análise ajustada } \\
\text { RP (IC95\%) }\end{array}$} \\
\hline & & & & \\
\hline \multirow[t]{3}{*}{1} & Sexo & & & \\
\hline & Masculino & 17,9 & 1,00 & 1,00 \\
\hline & Feminino & 18,1 & $1,01(0,78 ; 1,31)$ & $1,09(0,84 ; 1,40)$ \\
\hline \multirow[t]{4}{*}{1} & Faixa etária & & & \\
\hline & $20-39$ & 23,9 & 1,00 & 1,00 \\
\hline & $40-59$ & 14,0 & $0,59(0,44 ; 0,79)$ & $0,65(0,47 ; 0,89)$ \\
\hline & $60 \mathrm{ou}+$ & 10,4 & $0,43(0,28 ; 0,68)$ & $0,50(0,32 ; 0,80)$ \\
\hline \multirow[t]{3}{*}{1} & Cor & & & \\
\hline & Branca & 16,9 & 1,00 & 1,00 \\
\hline & Outras & 23,8 & $1,41(0,94 ; 2,13)$ & $1,23(0,82 ; 1,84)$ \\
\hline \multirow[t]{3}{*}{1} & Estado civil & & & \\
\hline & Solteiro & 23,4 & $1,90(1,42 ; 2,53)$ & $1,50(1,11 ; 2,03)$ \\
\hline & Casado, separado, viúvo & 12,3 & 1,00 & 1,00 \\
\hline \multirow[t]{4}{*}{1} & Escolaridade (anos) & & & \\
\hline & 0 a 8 & 19,8 & $1,34(0,94 ; 1,91)$ & $1,62(1,16 ; 2,27)$ \\
\hline & 9 a 11 & 19,3 & $1,31(0,88 ; 1,95)$ & $1,32(0,90 ; 1,95)$ \\
\hline & $\geq 12$ & 14,8 & 1,00 & 1,00 \\
\hline \multirow[t]{4}{*}{1} & Índice de bens (tercis) & & & \\
\hline & 1 (menor) & 18,8 & $1,16(0,85 ; 1,57)$ & $0,85(0,63 ; 1,14)$ \\
\hline & 2 & 19,1 & $1,17(0,87 ; 1,58)$ & $0,95(0,71 ; 1,26)$ \\
\hline & 3 (maior) & 16,3 & 1,00 & 1,00 \\
\hline \multirow[t]{4}{*}{2} & Tabagismo & & & \\
\hline & Não fumante & 18,6 & 1,00 & 1,00 \\
\hline & Ex-fumante & 13,5 & $0,73(0,51 ; 1,03)$ & $0,84(0,58 ; 1,21)$ \\
\hline & Fumante & 21,9 & $1,18(0,86 ; 1,62)$ & $1,12(0,81 ; 1,54)$ \\
\hline \multirow[t]{3}{*}{2} & Consumo excessivo de álcool & & & \\
\hline & Não & 17,7 & 1,00 & 1,00 \\
\hline & Sim & 20,4 & $1,15(0,85 ; 1,58)$ & $1,05(0,75 ; 1,45)$ \\
\hline \multirow[t]{2}{*}{2} & Consumo de doces & & & \\
\hline & Não & 18,4 & 1,00 & 1,00 \\
\hline
\end{tabular}


Tabela 2. Continuação...

\begin{tabular}{|c|c|c|c|c|}
\hline \multirow{2}{*}{ Nível } & \multirow{2}{*}{ Variável } & \multirow{2}{*}{$\begin{array}{c}\text { Dor de dente } \\
(\%)\end{array}$} & \multirow{2}{*}{$\begin{array}{c}\text { Análise bruta } \\
\text { RP (IC95\%) } \\
\end{array}$} & \multirow{2}{*}{$\begin{array}{c}\text { Análise ajustada } \\
\text { RP (IC95\%) } \\
\end{array}$} \\
\hline & & & & \\
\hline & Sim & 17,3 & $0,94(0,74 ; 1,20)$ & $0,92(0,73 ; 1,17)$ \\
\hline \multirow[t]{3}{*}{2} & Consumo de refrigerantes & & & \\
\hline & Não & 14,4 & 1,00 & 1,00 \\
\hline & Sim & 22,3 & $1,54(1,18 ; 2,03)$ & $1,30(0,99 ; 1,71)$ \\
\hline \multirow[t]{3}{*}{3} & Número de vezes que escova os dentes por dia & & & \\
\hline & Até 2 & 16,9 & $0,91(0,68 ; 1,21)$ & $1,15(0,81 ; 1,65)$ \\
\hline & $\geq 3$ & 13,6 & 1,00 & 1,00 \\
\hline \multirow[t]{3}{*}{3} & Uso diário de fio dental & & & \\
\hline & Não & 19,2 & 1,00 & 1,00 \\
\hline & Sim & 15,2 & $0,79(0,58 ; 1,09)$ & $0,84(0,61 ; 1,16)$ \\
\hline \multirow[t]{3}{*}{3} & Consulta ao dentista no último ano & & & \\
\hline & Não & 16,6 & 1,00 & 1,00 \\
\hline & Sim & 18,8 & $1,14(0,87 ; 1,49)$ & $1,17(0,88 ; 1,55)$ \\
\hline \multirow[t]{4}{*}{3} & Tipo de serviço usado na última consulta & & & \\
\hline & Público & 20,7 & 1,00 & 1,00 \\
\hline & Particular & 17,6 & $0,85(0,62 ; 1,17)$ & $1,00(0,68 ; 1,44)$ \\
\hline & Convênio & 15,9 & $0,77(0,50 ; 1,17)$ & $0,92(0,59 ; 1,42)$ \\
\hline \multirow[t]{3}{*}{3} & Range os dentes & & & \\
\hline & Não & 17,1 & 1,00 & 1,00 \\
\hline & Sim & 25,9 & $1,51(1,04 ; 2,19)$ & $1,67(1,18 ; 2,38)$ \\
\hline \multirow[t]{3}{*}{3} & Uso de prótese & & & \\
\hline & Não & 19,3 & 1,00 & 1,00 \\
\hline & Sim (parcial) & 12,3 & $0,64(0,44 ; 0,93)$ & $0,69(0,46 ; 1,21)$ \\
\hline & IC95: Intervalo de C & nfiança de 95\%. & & \\
\hline
\end{tabular}

\section{DISCUSSÃO}

No presente estudo de base populacional, na amostra representativa de adultos e idosos da cidade de Rio Grande-RS, um em cada cinco indivíduos apresentou dor dentária. A prevalência de dor de origem dentária encontrada no estudo foi menor quando comparada às prevalências encontradas no último inquérito nacional de saúde bucal realizado no ano de 2010 , indicando $27,5 \%^{2}$. Essa diferença pode ser justificada pelas dimensões continentais do Brasil, que ainda enfrenta problemas de acesso aos serviços de saúde bucal, principalmente em regiões mais distantes e vulneráveis do país ${ }^{11}$, que também são aquelas que apresentam as maiores prevalências de agravos bucais que acarretam dor dentária ${ }^{2}$. Entretanto, nosso resultado foi semelhante à frequência de dor dentária de 15,2\% encontrada na pesquisa telefônica realizada em 2009 - em todas as capitais brasileiras e no Distrito Federal - Vigitel ${ }^{6}$ - e à encontrada em estudo de base populacional realizado em Florianópolis-SC, em que a prevalência de dor dentária foi de 17,5\%7 . Em estudos de base populacional realizados em outros países de renda média e baixa, comparáveis ao Brasil, os resultados foram bem distintos entre si, sendo que a menor prevalência de dor dentária de $10,0 \%$ foi encontrada em uma amostra de 34.843 adultos colombianos $^{12}$ e a maior foi de $55,1 \%$ entre adultos iranianos ${ }^{13}$.

A prevalência de dor de dente foi menor para adultos de meia idade e idosos em comparação aos adultos jovens, neste estudo. Em Lages, pesquisa realizada por Kuhnen et al. ${ }^{14}$, em cidade catarinense de mesmo porte populacional que Rio Grande-RS, também encontrou que quanto maior a idade, menor a prevalência de relato de dor dentária nos últimos seis meses ${ }^{14}$. Além disso, na literatura internacional, essa associação inversa entre idade e relato de dor dentária também pode ser observada ${ }^{8,12,15}$. Provavelmente, isso pode ser explicado pelo fato de que indivíduos com idades mais avançadas sofrem com a herança de modelo odontológico curativo-mutilador ${ }^{16}$, em 
que se observam altas prevalências de edentulismo e perdas dentárias importantes ${ }^{17}$; por conseguinte, os idosos relatam menos dor dentária devido à ausência de dentes. Por outro lado, os mais jovens têm mais dentes presentes na boca que estão suscetíveis à cárie dentária e, por isso, apresentam maior chance de experimentar dor de origem dentária ${ }^{14}$. Dessa forma, conforme a literatura, o grupo etário que mais apresenta dor dentária é aquele com idade para estar inserido no mercado de trabalho ${ }^{5}$. A dor dentária em uma população de idade economicamente ativa pode gerar prejuízos, porque um trabalhador com dor de dente é mais provável faltar ao serviço por motivos odontológicos 4 .

Com relação ao estado civil, a dor dentária foi maior para os indivíduos solteiros, no presente estudo. Este resultado pode ser explicado pelo fato de que normalmente as pessoas casadas têm melhores indicadores de saúde quando comparadas às pessoas com outros estados civis, provavelmente em decorrência do maior suporte social e cuidado que essas pessoas recebem dos seus cônjuges ${ }^{18}$.

Em grande parte da literatura que aborda a temática, a dor dentária esteve associada ao menor nível de escolaridade $4,7,12,14$, o que é consistente com os achados do presente estudo. A escolaridade está relacionada ao conhecimento e às atitudes que os indivíduos têm sobre a própria saúde. Dessa forma, aqueles menos escolarizados podem deixar de adotar comportamentos saudáveis ${ }^{19}$ e, consequentemente, ter mais dor de dente. Ademais, em nosso estudo, a renda não apresentou associação estaticamente significativa com o relato de dor dentária e estes resultados sugerem que a dor dentária é mais uma questão de falta de conhecimento sobre hábitos saudáveis de saúde bucal para prevenir a dor do que a ausência de recursos financeiros para uma correta prevenção de agravos bucais.

Há uma ampla gama de estudos que buscam explicar a associação entre o bruxismo com as disfunções temporomandibulares, os quais foram sintetizados pela revisão sistemática realizada por Jiménez-Silva et al. ${ }^{20}$. Contudo, apenas um estudo investigou a associação entre bruxismo e a dor dentária, e encontrou diferença estatística significativa ${ }^{21}$, assim como no presente estudo. A hipótese dessa associação baseia-se no fato de que indivíduos que desenvolvem o hábito parafuncional de ranger os dentes podem apresentar danos ou desgastes dentários, que levam à maior probabilidade da ocorrência de dor dentária. No entanto, essa associação pode estar sujeita ao viés de causalidade reversa. Por essa razão, mais estudos para avaliar essa associação são recomendados.

Este estudo encontrou uma associação limítrofe da dor de dente com o consumo de refrigerantes após o ajuste para os possíveis confundidores. Este resultado pode indicar que houve falta de poder do estudo para encontrar essa associação, uma vez que, em um estudo sobre os fatores associados à dor de dente em adolescentes brasileiros, utilizando os dados de 54.985 participantes da Pesquisa Nacional de Saúde do Escolar (PeNSE) em 2009, uma maior prevalência de dor dentária foi observada entre os consumidores regulares de refrigerantes ${ }^{22}$. Neste sentido, o consumo regular de refrigerante é um hábito não saudável que influencia no aparecimento de outras doenças, como obesidade, o que pode ser modificado para também ajudar a diminuir a probabilidade de dor dentária.

Por fim, o presente estudo apresenta algumas limitações. Primeiramente, o delineamento transversal utilizado não permite estabelecer causalidade entre as exposições e o desfecho. Além disso, variáveis clínicas de saúde bucal que podem influenciar a dor dentária não foram medidas, como a avaliação da cárie dentária, da doença periodontal e do traumatismo dentário. Por outro lado, o estudo possui fortalezas que validam os resultados encontrados, como a representatividade da amostra estudada. Assim, os resultados obtidos neste estudo fornecem informações relevantes para a prevenção da dor dentária. 


\section{CONCLUSÃO}

Diante dos resultados do presente estudo, maior atenção deve ser dada aos solteiros, menos escolarizados, que rangem os dentes e adultos jovens, para prevenir a dor dentária e os impactos que ela causa na vida dos indivíduos. Por fim, recomenda-se que novas pesquisas com delineamento longitudinal sobre essa temática sejam realizadas para elucidar algumas associações observadas em estudos transversais.

\section{REFERÊNCIAS}

1. Goes P, Kosminsky M, Siqueira J, Ribeiro M. Epidemiologia da saúde bucal. Rio de Janeiro: Editora Guanabara Koogan; 2006.

2. Brasil. Ministério da Saúde. Secretaria de Atenção à Saúde. Secretaria de Vigilância em Saúde. SB Brasil 2010: Pesquisa Nacional de Saúde Bucal: resultados principais. Brasília: Ministério da Saúde; 2012.

3. Svensson L, Hakeberg M, Wide U. Dental pain and oral health-related quality of life in individuals with severe dental anxiety. Acta Odontol Scand. 2018 Aug;76(6):401-6. http://dx.doi.org/10.1080/00016357.2018.1473892. PMid:29782197.

4. Miotto MHMB, Silotti JCB, Barcellos LA. Dor dentária como motivo de absenteísmo em uma população de trabalhadores. Cien Saude Colet. 2012;17(5):1357-63. http://dx.doi.org/10.1590/S1413 81232012000500029. PMid:22634828.

5. Barcellos LA, Uliana AC, Miotto MHMB, Almeida ER. Dental pain prevalence among health care personnel. Rev Dor. 2015 Jun;16(2):119-23. http://dx.doi.org/10.5935/1806-0013.20150023.

6. Peres MA, Iser BPM, Peres KG, Malta DC, Antunes JLF. Desigualdades contextuais e individuais da prevalência de dor dentária em adultos e idosos no Brasil. Cad Saude Publica. 2012;28(Suppl):s114-23. http://dx.doi.org/10.1590/S0102-311X2012001300012. PMid:22714960.

7. Constante HM, Peres MA, Schroeder FC, Bastos JL. Mediators between education and dental pain: a cross-sectional study to assess the role of dental services utilization. Eur J Oral Sci. 2016 Feb;124(1):62-7. http://dx.doi.org/10.1111/eos.12242. PMid:26718615.

8. Momeni Z, Pakdaman A, Asadi-Lari M, Vaez-Mahdavi MR, Shamshiri AR, Hessari H. Dental pain and its determinants in an adult population in Tehran, Iran, Urban HEART-2. Community Dent Health. 2016 Sep;33(3):232-6. http://dx.doi.org/10.1922/CDH_3818Momeni05. PMid:28509520.

9. Instituto Brasileiro de Geografia e Estatistica - IBGE. Censo demográfico 2010. Rio de Janeiro: IBGE; 2011.

10. Dumith SC, Paulitsch RG, Carpena MX, Muraro MFR, Simões MO, Machado KP, et al. Planejamento e execução de um inquérito populacional de saúde por meio de consórcio de pesquisa multidisciplinar. Sci Med. (Porto Alegre, Online); 2018 Jul-Set;28(3):ID30407.

11. Peres MA, Iser BPM, Boing AF, Yokota RTC, Malta DC, Peres KG. Desigualdades no acesso e na utilização de serviços odontológicos no Brasil: análise do Sistema de Vigilância de Fatores de Risco e Proteção para Doenças Crônicas por Inquérito Telefônico (VIGITEL 2009). Cad Saude Publica. 2012;28(Suppl):s90-100. http://dx.doi.org/10.1590/S0102-311X2012001300010. PMid:22714973.

12. Ardila CM, Agudelo-Suarez AA. Association between dental pain and caries: a multilevel analysis to evaluate the influence of contextual and individual factors in 34843 adults. J Investig Clin Dent. 2016 Nov;7(4):410-6. http://dx.doi.org/10.1111/jicd.12168. PMid:26084783.

13. Kakoei S, Parirokh M, Nakhaee N, Jamshidshirazi F, Rad M, Kakooei S. Prevalence of toothache and associated factors: a population-based study in southeast iran. Iran Endod J. 2013;8(3):123-8. PMid:23922574. 
14. Kuhnen M, Peres MA, Masiero AV, Peres KG. Toothache and associated factors in Brazilian adults: a cross-sectional population-based study. BMC Oral Health. 2009 Feb;9(1):7. http://dx.doi.org/10.1186/1472-6831-9-7. PMid:19243630.

15. Pau A, Croucher RE, Marcenes W. Demographic and socio-economic correlates of dental pain among adults in the United Kingdom, 1998. Br Dent J. 2007 May;202(9):E21, discussion 548-9. http://dx.doi.org/10.1038/bdj.2007.171. PMid:17322865.

16. Moreira RS, Nico LS, Tomita NE, Ruiz T. A saúde bucal do idoso brasileiro: revisão sistemática sobre o quadro epidemiológico e acesso aos serviços de saúde bucal. Cad Saude Publica. 2005 Dec;21(6):166575. http://dx.doi.org/10.1590/S0102-311X2005000600013. PMid:16410850.

17. Cardoso M, Balducci I, Telles DM, Lourenço EJV, Nogueira L Jr. Edentulism in Brazil: trends, projections and expectations until 2040. Cien Saude Colet. 2016 Apr;21(4):1239-46. http://dx.doi.org/10.1590/1413-81232015214.13672015. PMid:27076022.

18. Rendall MS, Weden MM, Favreault MM, Waldron $\mathrm{H}$. The protective effect of marriage for survival: a review and update. Demography. 2011 May;48(2):481-506. http://dx.doi.org/10.1007/s13524-0110032-5. PMid:21526396.

19. Ferreira CO, Antunes JLF, Andrade FB. Factors associated with the use of dental services by elderly Brazilians. Rev Saude Publica. 2013 Dec;47(Suppl 3):90-7. http://dx.doi.org/10.1590/S00348910.2013047004721. PMid:24626585.

20. Jiménez-Silva A, Peña-Durán C, Tobar-Reyes J, Frugone-Zambra R. Sleep and awake bruxism in adults and its relationship with temporomandibular disorders: a systematic review from 2003 to 2014. Acta Odontol Scand. 2017 Jan;75(1):36-58. http://dx.doi.org/10.1080/00016357.2016.1247465. PMid:27796166.

21. de Siqueira SRDT, Vilela TT, Florindo AA. Prevalence of headache and orofacial pain in adults and elders in a Brazilian community: an epidemiological study. Gerodontology. 2015 Jun;32(2):123-31. http://dx.doi.org/10.1111/ger.12063. PMid:23789817.

22. Freire MCM, Leles CR, Sardinha LMV, Paludetto M Jr, Malta DC, Peres MA. Dor dentária e fatores associados em adolescentes brasileiros: a Pesquisa Nacional de Saúde do Escolar (PeNSE), Brasil, 2009. Cad Saude Publica. 2012;28(Suppl):s133-45. http://dx.doi.org/10.1590/S0102$311 X 2012001300014$.

\section{CONFLITOS DE INTERESSE}

Os autores declaram não haver conflitos de interesse.

\section{*AUTOR PARA CORRESPONDÊNCIA}

Mariana Silveira Echeverria, UFPel - Universidade Federal de Pelotas, R. Marechal Deodoro, 1160, 3ํㅜㄹ piso, 96020-220 Pelotas - RS, Brasil, e-mail: mari_echeverria@hotmail.com

Recebido: Maio 28, 2020

Aprovado: Agosto 6, 2020 